\title{
ON THE KOBAYASHI AND CARATHÉODORY DISTANCES OF BOUNDED SYMMETRIC DOMAINS
}

\author{
By Yoshinisa KuBota
}

1. Let $U$ denote the unit disk in the complex plane $C$ and let $\rho$ be the Poincaré distance in $U . \rho$ is given by

$$
\rho(z, w)=\frac{1}{2} \log \frac{1+\left|\frac{z-w}{1-\bar{w} z}\right|}{1-\left|\frac{z-w}{1-\bar{w} z}\right|} \quad(z, w \in U) .
$$

Since any automorphism $\phi$ of $U$ with $\phi(w)=0$ is given by

$$
\phi(z)=e^{i \theta} \frac{z-w}{1-\bar{w} z}
$$

with some $\theta \in \boldsymbol{R}$, the distance $\rho$ is also represented in the following:

$$
\begin{gathered}
\rho(z, w)=\inf \left\{\frac{1}{2} \log \frac{1+r}{1-r}: 0<r<1 \text { and } r U \ni \phi(z)\right. \text { for some } \\
\phi \in \operatorname{Aut}(U) \text { with } \phi(w)=0\},
\end{gathered}
$$

where $\operatorname{Aut}(U)$ denotes the group of automorphisms of $U$ and $r U$ denotes the set $\{z \in \boldsymbol{C}:|z|<r\}$. Furthermore, we have

$$
\{z \in U: \rho(0, z)<\alpha\}=r U, \quad r=\frac{e^{2 \alpha}-1}{e^{2 \alpha}+1} .
$$

In this note we show that the Kobayashi-Carathéodory distance of a bounded symmetric domain has the same property. (It is known that, for a bounded symmetric domain, the Kobayashi distance and the Carathéodory distance coincide [3]). Namely, let $D$ be a bounded symmetric domain given in a canonical realization in the complex $N$-space $\boldsymbol{C}^{N}$, and let $k_{D}$ be the Kobayashi-Carathéodory distance of $D$. Then we get

$$
\begin{gathered}
k_{D}(z, w)=\inf \left\{\frac{1}{2} \log \frac{1+r}{1-r}: 0<r<1 \text { and } r D \ni \phi(z)\right. \text { for some } \\
\phi \in \operatorname{Aut}(D) \text { with } \phi(w)=0\},
\end{gathered}
$$

Received May 12, 1988, Revised July 8, 1988 
and

$$
\left\{z \in D: k_{D}(0, z)<\alpha\right\}=r D, \quad r=\frac{e^{2 \alpha}-1}{e^{2 \alpha}+1},
$$

where $\operatorname{Aut}(D)$ denotes the group of automorphisms of $D$ and $r D$ denotes the set $\{r z: z \in D\}$ in $\boldsymbol{C}^{N}$.

2. In this section we consider domains $D$ in $C^{N}$ satisfying the following conditions :

(a) $D$ is homogeneous,

(b) $D$ is bounded,

(c) $D$ is convex and contains the origin 0 .

The following facts follow from (b) and (c).

(i) If $0<r<1$, then $\overline{r D}$ is a compact subset of $D$.

(ii) If $0<r_{1}<r_{2}<1$, then $\overline{r_{1} D} \subset r_{2} D$.

(iii) If $K$ is a compact subset of $D$, then $K \subset r D$ for some $r$ with $0<r<1$. Here $\overline{r D}$ denotes the closure of $r D$ in $\boldsymbol{C}^{N}$.

(a) and (iii) enable us to introduce the functions $d_{w}^{D}: D \rightarrow[0, \infty)$ (where $w \in$ $D)$ and $d_{D}: D \times D \rightarrow[0, \infty)$;

$$
\begin{aligned}
& d_{w}^{D}(z)=\inf \left\{\frac{1}{2} \log \frac{1+r}{1-r}: 0<r<1 \text { and } r D \ni \phi(z)\right. \text { for some } \\
& \qquad \phi \in \operatorname{Aut}(D) \text { with } \phi(w)=0\}, \\
& d_{D}(z, w)=\min \left\{d_{w}^{D}(z), d_{z}^{D}(w)\right\}
\end{aligned}
$$

We note that, for the unit disk $U$ in $\boldsymbol{C}$, we have

$$
d_{w}^{U}(z)=d_{z}^{U}(w)=d_{U}(z, w)=\rho(z, w) \quad(z, w \in U) .
$$

Proposition 1. The functions $d_{w}^{D}$ and $d_{D}$ have the following properties:

(1) $d_{w}^{D}(z) \geqq 0$, and $d_{w}^{D}(z)=0$ implies $z=w ; d_{D}(z, w) \geqq 0$, and $d_{D}(z, w)=0$ implies $z=w$.

(2) $d_{D}(z, w)=d_{D}(w, z)$.

(3) If $z \rightarrow \partial D$, then $d_{w}^{D}(z) \rightarrow \infty, d_{z}^{D}(w) \rightarrow \infty$ and $d_{D}(z, w) \rightarrow \infty$, where $\partial D$ denotes the boundary of $D$.

(4) If $\psi \in \operatorname{Aut}(D)$, then $d_{\phi(w)}^{D}(\psi(z))=d_{w}^{D}(z)$ and $d_{D}(\psi(z), \phi(w))=d_{D}(z, w)$.

(5) If $w \in D$ and $\alpha>0$, then

$$
\left\{z \in D: d_{w}^{D}(z)<\alpha\right\}=\bigcup_{\psi \in \operatorname{Aut}(D), \psi(0)=w} \psi(r D), \quad r=\frac{e^{2 \alpha}-1}{e^{2 \alpha}+1} .
$$

Proof. (2), (4) and (5) are immediate consequences of the definitions of $d_{w}^{D}$ and $d_{D}$. 
To prove (1) let $z$ and $w$ be distinct points in $D$. Suppose there exists a sequence $\left\{\phi_{n}\right\}$ of elements of Aut $(D)$ such that $\phi_{n}(w)=0$ for all $n$ and $\phi_{n}(z) \rightarrow 0$ as $n \rightarrow \infty$. Since $D$ is bounded, $\left\{\phi_{n}\right\}$ is a normal family. Hence we may suppose that $\left\{\phi_{n}\right\}$ converges, uniformly on every compact subset of $D$, to a holomorphic mapping $\phi: D \rightarrow C^{N}$. Since $\phi(w)=\lim _{n \rightarrow \infty} \phi_{n}(w)=0 \in D, \phi(D) \not \subset \partial D$ and so $\phi \in \operatorname{Aut}(D)$ ([5] p. 78). Thus we are led to a contradiction that $0=\phi(z) \neq \phi(w)=0$. Hence there exists a positive number $\delta$ such that $\delta B_{N} \not \nexists \phi(z)$ for all $\phi \in A u t(D)$ with $\phi(w)=0$. Here $B_{N}$ denotes the unit ball in $C^{N}$. Since $D$ is bounded, $\delta B_{N} \supset r_{0} D$ for some $r_{o}$ with $0<r_{0}<1$. Now it follows from (ii) that $d_{w}^{D}(z) \geqq \frac{1}{2} \log \frac{1+r_{0}}{1-r_{0}}$ $>0$, and (1) follows.

Next we prove (3). Suppose that there exists a positive number $\alpha$ such that, for any compact subset $K$ of $D$, there is a point $z \notin K$ with $d_{z}^{D}(w)<\alpha$. Then we can choose a sequence $\left\{z^{(n)}\right\}$ of points in $D$ such that $\left\{z^{(n)}\right\}$ tends to a boundary point $\zeta$ and such that $d_{z(n)}^{D}(w)<\alpha$ for all $n$. By the definition of $d_{z(n)}^{D}(w)$, we can choose sequences $\left\{r_{n}\right\}$ and $\left\{\phi_{n}\right\}$ such that

and

$$
0<r_{n}<1, \quad \frac{1}{2} \log \frac{1+r_{n}}{1-r_{n}}<\alpha
$$

$$
\phi_{n} \in \operatorname{Aut}(D), \quad \phi_{n}\left(z^{(n)}\right)=0, \quad \phi_{n}(w) \in r_{n} D .
$$

Since $\left\{\phi_{n}^{-1}\right\}$ is a normal family and since $\phi_{n}(w) \in \overline{r D}$ where $r=\left(e^{2 \alpha}-1\right) /\left(e^{2 \alpha}+1\right)$, we may assume that $\left\{\phi_{n}^{-1}\right\}$ converges, uniformly on every compact subset of $D$, to a holomorphic mapping $\phi: D \rightarrow C^{N}$ and that $\phi_{n}(w) \rightarrow w^{*} \in \overline{r D}$. Hence we have

$$
\left\|\phi\left(w^{*}\right)-w\right\| \leqq\left\|\phi\left(w^{*}\right)-\phi\left(\phi_{n}(w)\right)\right\|+\left\|\phi\left(\phi_{n}(w)\right)-\phi_{n}^{-1}\left(\phi_{n}(w)\right)\right\| \longrightarrow 0 \quad \text { as } n \rightarrow \infty,
$$

where \|\| denotes the euclidean norm in $\boldsymbol{C}^{N}$, then $\phi\left(w^{*}\right)=w$. This implies that $\phi \in \operatorname{Aut}(D)\left([5]\right.$ p. 78). But this contradicts that $\phi(0)=\lim _{n \rightarrow \infty} \phi_{n}^{-1}(0)=\lim _{n \rightarrow \infty} z^{(n)}$ $=\zeta \in \partial D$. Thus we conclude that $d_{z}^{D}(w) \rightarrow \infty$ if $z \rightarrow \partial D$. Similarly we can prove that $d_{w}^{D}(z) \rightarrow \infty$ if $z \rightarrow \partial D$.

The function $d_{D}$ may not satisfy the triangle inequality. Following Kobayashi [2], we introduce $d_{D}$ by setting

$$
d_{D}(z, w)=\inf \sum_{j=0}^{k-1} d_{D}\left(z^{(j)}, z^{(\jmath+1)}\right),
$$

where the infimum is taken over all finite sequences $\left\{z^{(0)}, z^{(1)}, \cdots, z^{(k)}\right\}$ with $z^{(0)}=z$ and $z^{(k)}=w$. Then $d_{D}$ is a pseudodistance on $D$.

Next we consider domains $D$ which satisfy conditions (a), (b), (c) and an additional condition

(d) $D$ is circular.

The following lemma tells us that $d_{w}^{D}(z)$ decreases under holomorphic mappings:

LemMa 1. Let $D_{1}$ and $D_{2}$ be domains in $\boldsymbol{C}^{N_{1}}$ and $\boldsymbol{C}^{N_{2}}$, respectively, which 
satisfy conditions (a) (d). If $F: D_{1} \rightarrow D_{2}$ is a holomorphic mapping, then

$$
d_{F^{2}(w)}^{D_{2}}(F(z)) \leqq d_{w}^{D_{1}}(z) \quad\left(z, w \in D_{1}\right) .
$$

Proof. We shall first prove the inequality

$$
d_{0}^{D_{2}}(G(z)) \leqq d_{0}^{D_{1}}(z) \quad\left(z \in D_{1}\right)
$$

for a holomorphic mapping $G: D_{1} \rightarrow D_{2}$ with $G(0)=0$. Let $z \in D_{1}$ and $d_{0}^{D_{1}}(z)<\alpha$. Then there exists an $r, 0<r<1$, such that $\frac{1}{2} \log \frac{1+r}{1-r}<\alpha$ and $r D_{1} \ni \phi(z)$ for some $\phi \in \operatorname{Aut}\left(D_{1}\right)$ with $\phi(0)=0$. Put $H=G \circ \phi^{-1}$. Since $H: D_{1} \rightarrow D_{2}$ is a holomorphic mapping with $H(0)=0$ and since $D_{1}$ and $D_{2}$ satisfy conditions (b) (d), we have $H\left(r D_{1}\right) \subset r D_{2}$ ([6] p. 161). Hence $G(z)=H(\phi(z)) \in r D_{2}$, and therefore $d_{0}^{D_{2}}(G(z))<\alpha$. Thus we have

$$
d_{0}^{D_{2}}(G(z)) \leqq d_{0}^{D_{1}}(z) \quad\left(z \in D_{1}\right) .
$$

From this inequality we have, for any $z, w \in D_{1}$,

$$
d_{F^{2}(w)}^{D_{2}}(F(z))=d_{0}^{D_{2}}\left(\left(\psi_{2} \circ F \circ \psi_{1}^{-1}\right)\left(\psi_{1}(z)\right)\right) \leqq d_{0}^{D_{1}}\left(\psi_{1}(z)\right)=d_{w^{1}}^{D_{1}}(z)
$$

by taking $\phi_{1} \in \operatorname{Aut}\left(D_{1}\right)$ and $\phi_{2} \in \operatorname{Aut}\left(D_{2}\right)$ with $\phi_{1}(w)=0, \phi_{2}(F(w))=0$.

If $D$ is a domain in $\boldsymbol{C}^{N}$ which is holomorphically equivalent to a domain $\tilde{D}$ (i. e. there is a biholomorphic mapping of $D$ onto $\widetilde{D}$ ) satisfying conditions (a) (d), we define $d_{w}^{D}$ by

$$
d_{w}^{D}(z)=d_{\psi(w)}^{\tilde{D}}(\phi(z)),
$$

where $\phi$ is a biholomorphic mapping of $D$ onto $\widetilde{D}$. Note that this definition does not depend on choices of $\widetilde{D}$ and $\phi$. (It follows from Lemma 1). Hence we can also define $d_{D}$ and $d_{D}$ for $D$. The functions $d_{w}^{D}$ and $d_{D}$ have properties (1) (4) in Proposition 1. Further the following proposition is an immediate consequence of Lemma 1.

Proposition 2. Let $D_{1}$ and $D_{2}$ be domains in $C^{N_{1}}$ and $C^{N_{2}}$, respectively, which are holomorphically equivalent to domains satisfying conditions (a) $\sim(\mathrm{d})$. If $F: D_{1} \rightarrow D_{2}$ is a holomorphic mapping, then

and

$$
d_{F^{2}(w)}^{D_{2}}(F(z)) \leqq d_{w}^{D_{1}}(z) \quad\left(z, w \in D_{1}\right)
$$

$$
d_{D_{2}}(F(z), F(w)) \leqq d_{D_{1}}(z, w) \quad\left(z, w \in D_{1}\right) .
$$

Let $k_{D}$ and $c_{D}$ denote the Kobayashi pseudodistance and the Carathéodory pseudodistance of $D$, respectively. If $D$ is holomorphically equivalent to a domain satisfying conditions $(\mathrm{a}) \sim(\mathrm{d})$, then it follows from Proposition 2 that

$$
c_{D} \leqq d_{D} \leqq k_{D} .
$$


3. We shall now turn our attention to bounded symmetric domains. It is known that every bounded symmetric domain is holomorphically equivalent to a domain in $\boldsymbol{C}^{N}$ satisfying (a) (d) [4]. (Conversely, every domain in $\boldsymbol{C}^{N}$ satisfying conditions (a), (c), (d) is symmetric). Hence $d_{w}^{D}, d_{D}$ and $d_{D}$ are defined on bounded symmetric domains $D$. In this section we shall show that, for a bounded symmetric domain $D, d_{w}^{D}, d_{D}$ and $d_{D}$ coincide with $k_{D}$ and $c_{D}$. Our proof will follow Kobayashi's argument ([3] p. 52) which was used to prove that $k_{D}=c_{D}$ for bounded symmetric domains $D$.

LeMma 2. Let $U^{N}$ be the unit polydisk in $\boldsymbol{C}^{N}$. Then

$$
d_{w}^{U N}(z)=d_{z}^{U^{N}}(w)=\max \left\{\rho\left(z_{\jmath}, w_{j}\right): j=1, \cdots, N\right\}
$$

where $z=\left(z_{1}, \cdots, z_{N}\right)$ and $w=\left(w_{1}, \cdots, w_{N}\right)$.

Proof. Let $z^{*}=\left(z_{1}^{*}, \cdots, z_{N}^{*}\right)$ and $w^{*}=\left(w_{1}^{*}, \cdots, w_{N}^{*}\right)$ be points in $U^{N}$ and let $d_{w^{*}}^{U^{N}}\left(z^{*}\right)<\alpha$. Then $\frac{1}{2} \log \frac{1+r}{1-r}<\alpha$ and $r U^{N} \ni \phi\left(z^{*}\right)$ for some $r, 0<r<1$, and for some $\phi \in \operatorname{Aut}\left(U^{N}\right)$ with $\phi\left(w^{*}\right)=0$. Now $\phi$ has a form

$$
\begin{aligned}
& \phi(z)=\left(\phi_{1}\left(z_{p(1)}\right), \cdots, \phi_{N}\left(z_{p(N)}\right)\right), \quad z=\left(z_{1}, \cdots, z_{N}\right), \\
& \phi_{j}(\zeta)=\varepsilon_{j} \frac{\zeta-w_{p(j)}^{*}}{1-w_{p(j)}^{*} \zeta} \quad(j=1, \cdots, N ; \zeta \in U),
\end{aligned}
$$

where $\varepsilon_{j} \in C,\left|\varepsilon_{j}\right|=1$ and $p$ is a permutation of the integers from 1 to $N$ ([5] p. 68). Since $\phi_{j} \in \operatorname{Aut}(U), \phi_{j}\left(w_{p(j)}^{*}\right)=0$ and $\phi_{j}\left(z_{p(j)}^{*}\right) \in r U$, we have

$$
\rho\left(z_{\jmath}^{*}, w_{\jmath}^{*}\right)=d_{w_{j}^{*}}^{U}\left(z_{\jmath}^{*}\right) \leqq \frac{1}{2} \log \frac{1+r}{1-r}<\alpha \quad(j=1, \cdots, N) .
$$

Since $\alpha$ was arbitrary, we have

$$
\max \left\{\rho\left(z_{\jmath}^{*}, w_{\jmath}^{*}\right): j=1, \cdots, N\right\} \leqq d_{w^{*}}^{U N}\left(z^{*}\right) .
$$

To prove the inequality in the opposite direction, let $\rho\left(z_{j}^{*}, w_{3}^{*}\right)<\alpha$ for all $j$. Then we can choose an $r, 0<r<1$, and $\phi_{j} \in \operatorname{Aut}(U), j=1, \cdots, N$, such that $\frac{1}{2} \log \frac{1+r}{1-r}<\alpha$ and $\phi_{j}\left(z_{\jmath}^{*}\right) \in r U$ and $\phi_{j}\left(w_{\jmath}^{*}\right)=0$. Put

$$
\phi(z)=\left(\phi_{1}\left(z_{1}\right), \cdots, \phi_{N}\left(z_{N}\right)\right), \quad z=\left(z_{1}, \cdots, z_{N}\right) .
$$

Then $\phi$ is an element of Aut $\left(U^{N}\right)$ satisfying $\phi\left(w^{*}\right)=0$ and $\phi\left(z^{*}\right) \in r U^{N}$. Hence

$$
d_{w^{*}}^{U N}\left(z^{*}\right) \leqq \frac{1}{2} \log \frac{1+r}{1-r}<\alpha .
$$

Thus we obtain that 


$$
d_{w^{*}}^{U^{N}}\left(z^{*}\right)=\max \left\{\rho\left(z_{\jmath}^{*}, w_{\jmath}^{*}\right): j=1, \cdots, N\right\} .
$$

Since $\rho\left(z_{\jmath}^{*}, w_{\jmath}^{*}\right)=\rho\left(w_{\jmath}^{*}, z_{\jmath}^{*}\right)$, we have also

$$
d_{w^{*}}^{U^{N}}\left(z^{*}\right)=d_{z^{*}}^{U^{N}}\left(w^{*}\right) \text {. }
$$

THEOREM 1. If $D$ is a bounded symmetric domain in $\boldsymbol{C}^{N}$, then

$$
k_{D}(z, w)=c_{D}(z, w)=d_{w}^{D}(z) \quad(z, w \in D) .
$$

Proof. It is known that $D$ is holomorphically equivalent to a domain $D^{*}$ which has the following properties:

( $\alpha) \quad D^{*} \cap C^{l}=U^{l}$ and $D^{*} \subset U^{N}$,

( $\beta)$ for any $z^{*}, w^{*} \in D^{*}$, there exists a $\phi \in \operatorname{Aut}\left(D^{*}\right)$ such that $\phi\left(w^{*}\right)=0$ and $\phi\left(z^{*}\right)=\zeta=\left(\zeta_{1}, \cdots, \zeta_{l}, 0, \cdots, 0\right)$ where $\left|\zeta_{j}\right|<1, j=1, \cdots, l$.

Here $l$ is the rank of $D$. Since the injections $U^{l} \rightarrow D^{*}$ and $D^{*} \rightarrow U^{N}$ are distancedecreasing (by Proposition 2), we have

$$
d_{0}^{U^{N}}(\zeta) \leqq d_{0}^{D^{*}}(\zeta) \leqq d_{0}^{U^{l}}\left(\zeta^{\prime}\right)
$$

where $\zeta=\left(\zeta^{\prime}, 0^{\prime \prime}\right), \zeta^{\prime}=\left(\zeta_{1}, \cdots, \zeta_{l}\right), 0^{\prime \prime}=(0, \cdots, 0)$. From Lemma 2 we have

$$
d_{0}^{U^{N}}(\zeta)=d_{0}^{U^{l}\left(\zeta^{\prime}\right)}=\max \left\{\boldsymbol{\rho}\left(\zeta_{j}, 0\right): j=1, \cdots, l\right\},
$$

and then we obtain

$$
d_{0}^{D^{*}}(\zeta)=\max \left\{\rho\left(\zeta_{\jmath}, 0\right): j=1, \cdots, l\right\} .
$$

It is also known that

$$
k_{D^{*}}(\zeta, 0)=c_{D^{*}}(\zeta, 0)=\max \{\rho(\zeta, 0): j=1, \cdots, l\}
$$

([3] p. 52). Hence it follows from $(\beta)$ that, for $z^{*}, w^{*} \in D^{*}$,

$$
d_{w^{*}}^{D^{*}}\left(z^{*}\right)=k_{D^{*}}\left(z^{*}, w^{*}\right)=c_{D^{*}}\left(z^{*}, w^{*}\right) .
$$

This implies that

$$
d_{w}^{D}(z)=k_{D}(z, w)=c_{D}(z, w) \quad(z, w \in D) .
$$

COROLlary 1. If $D$ is a bounded symmetric domain in $\boldsymbol{C}^{N}$, then

$$
d_{D}(z, w)=d_{D}(z, w)=d_{w}^{D}(z)=d_{z}^{D}(w) \quad(z, w \in D) .
$$

Every bounded symmetric domain in $C^{N}$ is holomorphically equivalent to a domain $D$ in $C^{N}$ which satisfies conditions (a) $\sim(\mathrm{d})$ and

(e) the isotropy group $K$ of 0 in $\operatorname{Aut}(D)$ acts by complex linear transformations.

We shall call such a domain $D$ a domain given in a canonical realization. The following is an immediate consequence of Theorem 1 and (5) in Proposition 1. 
THEOREM 2. Let $D$ be a bounded symmetric domain given in a canonical realization in $\boldsymbol{C}^{N}$. Then

$$
\begin{aligned}
k_{D}(z, w)=\inf \left\{\frac{1}{2} \log \frac{1+r}{1-r}:\right. & 0<r<1 \text { and } r D \ni \phi(z) \text { for some } \\
\phi & \in \operatorname{Aut}(D) \text { with } \phi(w)=0\}
\end{aligned}
$$

and

$$
\left\{z \in D: k_{D}(0, z)<\alpha\right\}=r D, \quad r=\frac{e^{2 \alpha}-1}{e^{2 \alpha}+1}
$$

COROLlARY 2. Let $D$ be a bounded symmetric domain given in a canonical realization in $\boldsymbol{C}^{N}$ and let $w \in \partial D$. If $0 \leqq t_{1}<t_{2}<t_{3}<1$, then

$$
k_{D}\left(t_{1} w, t_{3} w\right)=k_{D}\left(t_{1} w, t_{2} w\right)+k_{D}\left(t_{2} w, t_{3} w\right) .
$$

Proof. We shall first show that

$$
k_{D}(s w, t w)=k_{D}(0, t w)-k_{D}(0, s w)
$$

for $0<s<t<1$. Since $D$ satisfies conditions (c) and (d), $\{\zeta w: \zeta \in C,|\zeta|<1\} \subset D$. Hence

$$
f_{w}(\zeta)=\zeta w
$$

is a holomorphic mapping of $U$ into $D$, and so

$$
\begin{aligned}
k_{D}(s w, t w) & \leqq k_{U}(s, t)=\frac{1}{2} \log \frac{1+\frac{t-s}{1-s t}}{1-\frac{t-s}{1-s t}}=\frac{1}{2} \log \frac{1+t}{1-t}-\frac{1}{2} \log \frac{1+s}{1-s} \\
& =k_{D}(0, t w)-k_{D}(0, s w)
\end{aligned}
$$

by Theorem 2. But the triangle inequality yields

$$
k_{D}(s w, t w) \geqq k_{D}(0, t w)-k_{D}(0, s w) .
$$

Thus we obtain the equality

$$
k_{D}(s w, t w)=k_{D}(0, t w)-k_{D}(0, s w) \quad(0<s<t<1) .
$$

Using this equality we have, for $0 \leqq t_{1}<t_{2}<t_{3}<1$,

$$
\begin{aligned}
k_{D}\left(t_{1} w, t_{3} w\right) & =k_{D}\left(0, t_{3} w\right)-k_{D}\left(0, t_{1} w\right) \\
& =\left\{k_{D}\left(0, t_{3} w\right)-k_{D}\left(0, t_{2} w\right)\right\}+\left\{k_{D}\left(0, t_{2} w\right)-k_{D}\left(0, t_{1} w\right)\right\} \\
& =k_{D}\left(t_{2} w, t_{3} w\right)+k_{D}\left(t_{1} w, t_{2} w\right) .
\end{aligned}
$$




\section{REFERENCES}

[1] S. Helgason, Differential Geometry and Symmetric Spaces, Academic Press. New York, 1962.

[2] S. Kobayashi, Invariant distances on complex manifolds and holomorphic mappings, J. Math. Soc. Japan 19 (1967), 460-480.

[3] S. Kobayashi, Hyperbolic Manifolds and Holomorphıc Mappings, Marcel Dekker, New York, 1970.

[4] A. KorÁnYi AND J.A. Wolf, Realization of hermitıan symmetric spaces as generalized half-planes, Ann. of Math. 81 (1965), 265-288.

[5] R. Narasimhan, Several Complex Variables, University of Chicago Press, Chicago, 1971.

[6] W. Rudin, Function Theory in the Unit Ball of $\boldsymbol{C}^{n}$, Die Grundlehren der Math, Wissenschaften, Bd. 241, Springer-Verlag, Berlin, 1980.

Department of Mathematics

TOKYo GaKugEI UNIVERSITY

Koganei-Shi, TOKYo 184, JAPAN 